\title{
Immunohistopathological Effects of Combined Administration of Douvir-N and Folc Acid on the Liver and Some Biochemical Parameters in Albino Wistar Rats
}

\author{
Aniekan Imo Peter ${ }^{*}$, Gabriel Joseph Ekandem \\ Department of Anatomy, University of Uyo, Uyo, Nigeria \\ Email address: \\ aniekanpeter@uniuyo.edu.ng (A. I. Peter) \\ To cite this article: \\ Aniekan Imo Peter, Gabriel Joseph Ekandem. Immunohistopathological Effects of Combined Administration of Douvir-N and Folc Acid on \\ the Liver and Some Biochemical Parameters in Albino Wistar Rats. American Journal of BioScience. Vol. 3, No. 6, 2015 , pp. 197-202. \\ doi: $10.11648 /$ j.ajbio. 20150306.11
}

\begin{abstract}
Douvir-N is a combination of lamivudine, zidovudine and nevirapine used for the treatment of patients with Human Immunodeficiency Virus. The objective of this study was to investigate the effect of combined administration of Douvir-N and folic acid on the histology and some Biochemical parameters in the liver of Wistar rats. Forty adult albino Wistar rats were randomly divided into four groups of ten animals each. Group A served as control and were administered with $1 \mathrm{ml}$ of distilled water, group B animals were administered with $9.29 \mathrm{mg} / \mathrm{kg}$ body weight of Douvir- $\mathrm{N}$, Group C animals were administered with a combination of $9.29 \mathrm{mg} / \mathrm{kg}$ of Douvir-N and $0.07 \mathrm{mg} / \mathrm{kg}$ of folic acid. Animals in group D were administered with $0.07 \mathrm{mg} / \mathrm{kg}$ of folic acid. Animals were sacrificed after 30 days and dissected. The liver was removed and fixed in $10 \%$ buffered formaldehyde, processed and stained using Haematoxylin and Eosin staining method, carcino-embryonic antigen (CEA) and cytokeratin-7 (CK-7) immunochemistry methods. Stained slides were viewed using light microscope. Blood samples from each rat was collected using syringes and needles, The sera were extracted into fresh test tubes and stored in a refrigerator for analysis of aspartate aminotransaminase test (AST), alanine aminotransaminase test (ALT), alkaline phosphotase (ALP). The liver of Wistar rats administered with Douvir-N showed distortions in the liver with moderate dilatation of the sinusoidal spaces and nuclei pyknotic changes, with increased expression of CEA and CK7 in the groups treated with Douvir-N than the control groups. There was a significant increase in ALP in the Douvir-N groups. These changes were ameliorated when Douvir- $\mathrm{N}$ was combined with Folic acid. The findings suggest that Douvir-N can distort the cytoarchitecture and Biochemical parameters of the liver which could be ameliorated by co-administration with folic acid. Folic acid should be given as adjuvant drug to patients on Douvir-N therapy.
\end{abstract}

Keywords: Liver, Douvir-N, Folic Acid, Human Immunodeficiency Virus (HIV), Biochemical Parameters

\section{Introduction}

Douvir-N is an antiretroviral (ARV) drug that contains lamivudine, zidovudine and nevirapine. Belonging to the following two groups: nucleoside analogues (NRTIs, lamivudine and zidovudine) and non-nucleoside reverse transcriptase inhibitors (NNRTIs, nevirapine) [1]. It is used as antiretroviral combination therapy for the treatment of HIV infection [1].

The three medicines contained in Douvir-N can be used separately with other medicines for combination treatment of HIV infection or can be used together. The dose of each active ingredient in Douvir- $\mathrm{N}$ is the same as that recommended for the medicines when used separately. Douvir-N reduces the amount of HIV in the body [1]. It also increases CD4 cell counts $[1,2]$.

The triple drug therapy with Douvir-N makes it easier to take the medications regularly, which helps improve compliance and helps prevent resistance of HIV to individual drugs [3]. Modern combination therapy is highly effective and people with HIV on antiretroviral treatment could live for the rest of their lives without developing AIDS [2].

Despite these improvements, prolonged benefits of antiretroviral drugs are compromised by numerous side-effects, adverse clinical events and toxicities. All antiretroviral drugs can have both short-term and long-term adverse effects. The risk of specific side effects varies from drug to drug, from drug class to drug class and from patient to 
patient. Some of the clinical events include AIDS-related insulin resistance, lipodystropy syndrome, gastrointestinal symptoms, hyperglycemia $[4,5,6]$.

The most common and troublesome toxicities of Nucleoside Reverse Transcriptase Inhibitors (NRTIs) is hepatoxicity [6, 7]. Virtually every licensed antiretroviral medication has been associated with liver enzyme elevations [8]. Liver toxicity may also occur as a consequence of mitochondrial damage in patients receiving nucleosides analogues, particularly Zidovudine or Stavudine [9, 10]. Other detrimental effect of anti HIV drugs includes; allergies, hyperglycaemia, lactic acidosis, and gastrointestinal disorder [11], myelopathy, neuropathy, neurologic pain, changes in cognition and dementia [12].

Folate is a water-soluble B vitamin that is found naturally in foods such as fruits, dark green vegetables, potatoes, beans and yeast extracts. Folic acid is the synthetic form of folate found in dietary supplements and added to enriched flour and grain products [13]. Growing evidence suggests a potential role of folic acid in invivo and invitro antioxidants actions. When taken before conception, adequate use of folic acid reduces the incidence of Neural tube defects (NTDs) by $50-70 \%$ [14]. Neural tube defects are the results of abnormalities in neurulation $[15,16]$. Folate modulates a number of disorders as a result of its anti- apoptotic and anti-oxidative properties [17], this includes: cardiovascular diseases [18], neural tube and congenital defects [14], subfertility [14] and several malignancies like cancer of the colorectum, lungs, pancreas, esophagus, stomach, cervix, breast [20], neuroblastoma and leukemia [17]. A deficiency of folate may increase blood levels of homocysteine. It also impairs DNA synthesis and cell division. Folate supplementation has been shown to decrease homocysteine levels and to improve endothelial functions [21]. Folate supplementation is associated with improving memory deficits among cognitively impaired subjects. Higher folate intake is correlated with lower risks of Alzheimer's disease. $[22,23]$. The intake of folate has no known drug interaction with ARVs rather it enhances the delivery nanoformulated ritonavir (RTV)-boosted atazanavir when given to patients [24]. The aim of this study therefore was to investigate the immunohistopathological effects of Douvir-N and its combined administration with folic acid on the liver and some biochemical parameters in Albino Wistar rats.

\section{Methodology}

The drugs used in this study Douvir- $\mathrm{N}$ is a fixed dose combination of lamivudine, Zidovudine and Nevirapine. It was obtained from the University of Uyo Teaching Hospital (UUTH) Uyo, Nigeria. The drug was manufactured by Cipla pharmaceuticals of India. Folic acid was obtained from Top care pharmacy in Uyo. The drug was manufactured by Vitabiotics Nigeria limited. The drugs were prepared by grinding them using a mortar and pestle to powder form. It was then diluted with $100 \mathrm{ml}$ distilled water. Ethical approval was obtained from the Post Graduate School committee Faculty of Basic Medical Sciences, University of Uyo.
Forty adult albino Wistar rats weighing $260 \mathrm{~g} \pm 10 \mathrm{~g}$ body weights were obtained from the animal house of faculty of Basic Medical Sciences, University of Uyo, Nigeria. They were housed in cages and maintained under standard environmental conditions. The rats were fed with standard pellet diet and water. There were randomly divided into 4 groups (10 rats per group) and housed in cages. Douvir-N was administered twice daily, while folic acid was administered o daily. All drug administration was orally and lasted for 30 days. Group A was administered distilled water, they served as control. Group B Douvir-N was administered with $9.29 \mathrm{mg} / \mathrm{kg}$ body weight, Group $\mathrm{C}$ was administered a combination of Douvir-N $(9.29 \mathrm{mg} / \mathrm{kg})$ and folic acid $(0.07 \mathrm{mg} / \mathrm{kg})$, while group D was administered with folic acid alone $(0.07 \mathrm{mg} / \mathrm{kg})$.

The animals were sacrificed on the $31^{\text {st }}$ day after overnight fast using chloroform inhalation method. The abdominal cavity was dissected through a midline abdominal incision. The liver were extracted and rinsed in normal saline and fixed in $10 \%$ buffered formalin. They were then processed and stained with the Haematoxylin and Eosin staining method, carcino-embryonic antigen (CEA) and cytokeratin -7 \{CK-7) immunochemistry methods. Stained slides were viewed using light microscope. Blood samples from each rat were collected using syringes and needles and separated into sample bottles and allowed to stand for 30 minutes for clotting to take place and then centrifuged. The serum was extracted into fresh test tubes and stored in a refrigerator for analysis of aspartate aminotransaminase test (AST), alanine aminotransaminase test (ALT), alkaline phosphotase (ALP). Results were analyzed using one way Analysis of Variance (ANOVA) and post hoc test.

\section{Results}

The histomorphological features that are present in the various groups upon viewing under the light microscope are as follows:

The control group (A) administered with distill water and stained with $\mathrm{H} / \mathrm{E}$ showed normal liver architecture; the central vein $(\mathrm{V})$, hepatocytes plates $(\mathrm{H})$, sinusoidal spaces $(\mathrm{S})$ and nuclei $(\mathrm{N})$ are all normal, as shown in Fig. 1A. It also showed normal liver expression of CEA and Ck-7 by the hepatocytes as shown in Fig 2A and Fig 3A.

Group B administered with Douvir-N $9.29 \mathrm{mg} / \mathrm{kg}$ and stained with $\mathrm{H} / \mathrm{E}$ showed moderate distortion of liver cellular architecture; the central veins (V) and sinusoidal spaces (S) are dilated, hepatocytes plates $(\mathrm{H})$ are swollen and nuclei $(\mathrm{N})$ are pyknotic as shown in Fig 1B. It also showed moderate increased in the expression of CEA and $\mathrm{Ck}-7$ by the hepatocytes, as shown in Fig 2B and Fig 3B.

Group C administered with Douvir-N $9.29 \mathrm{mg} / \mathrm{kg}$ body weight and folic acid $(0.07 \mathrm{mg} / \mathrm{kg})$, showed mild distortion of liver cellular architecture; the central vein (V) are normal, hepatocytes plates $(\mathrm{H})$ are mildly swollen, sinusoidal spaces (S) are mildly dilated with slight area of nuclei $(\mathrm{N})$ karyolysis as shown in Fig 1C. It also showed mild increased in the expression of CEA and $\mathrm{Ck}-7$ by the hepatocytes as shown in 
Fig $2 \mathrm{C}$ and Fig $3 \mathrm{C}$.

Group D administered with folic acid $(0.07 \mathrm{mg} / \mathrm{kg})$, showed no distortion of liver cellular architecture; the central vein $(\mathrm{V})$ are normal, hepatocytes plates $(\mathrm{H})$ normal and the nuclei $(\mathrm{N})$ are normal as shown in Fig 1D. It also showed normal expression of CEA and Ck-7 by the hepatocytes as shown in Fig 2D and Fig 3D.
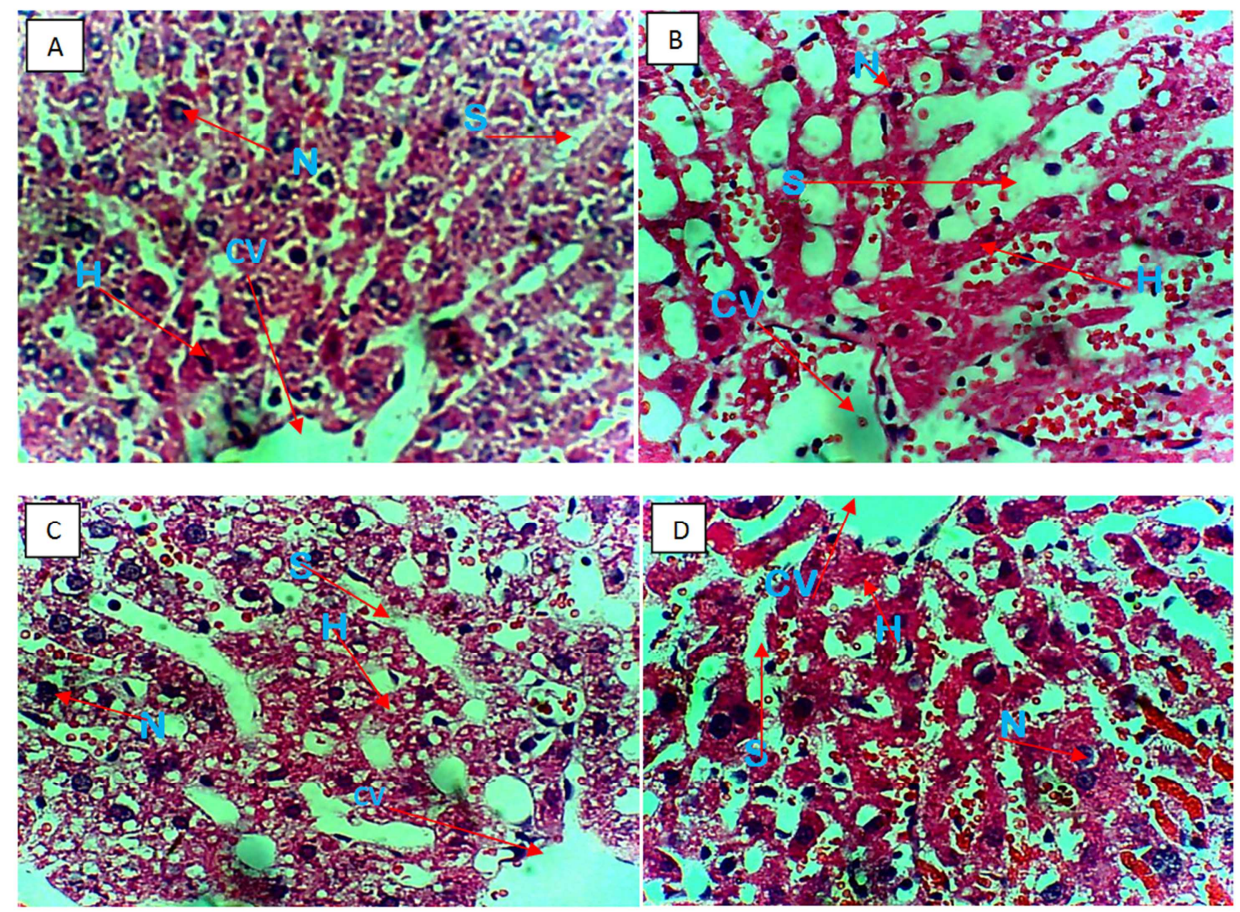

Figure 1. $(A, B, C, D): 1 A$, Micrograph of the liver of control rat administered with distill water $H \& E x 400.1 B$ Micrograph of the liver of a rat administered with $9.29 \mathrm{mg} / \mathrm{kg}$ body weight of Douvir-N H \& Ex400. 1C: Micrograph of the liver of a rat administered with $9.29 \mathrm{mg} / \mathrm{kg}$ body weight of Douvir-Nand $0.07 \mathrm{mg} / \mathrm{kg}$ of folic acid $H \& E x 400$. 1D: Micrograph of the liver of a rat administered with $0.07 \mathrm{mg} / \mathrm{kg}$ body weight of folic acid $H \&$ Ex $100 x 400$. All showing hepatocytes (H), central vein $(C V)$, nucleus $(N)$, sinusoidal spaces $(S)$.
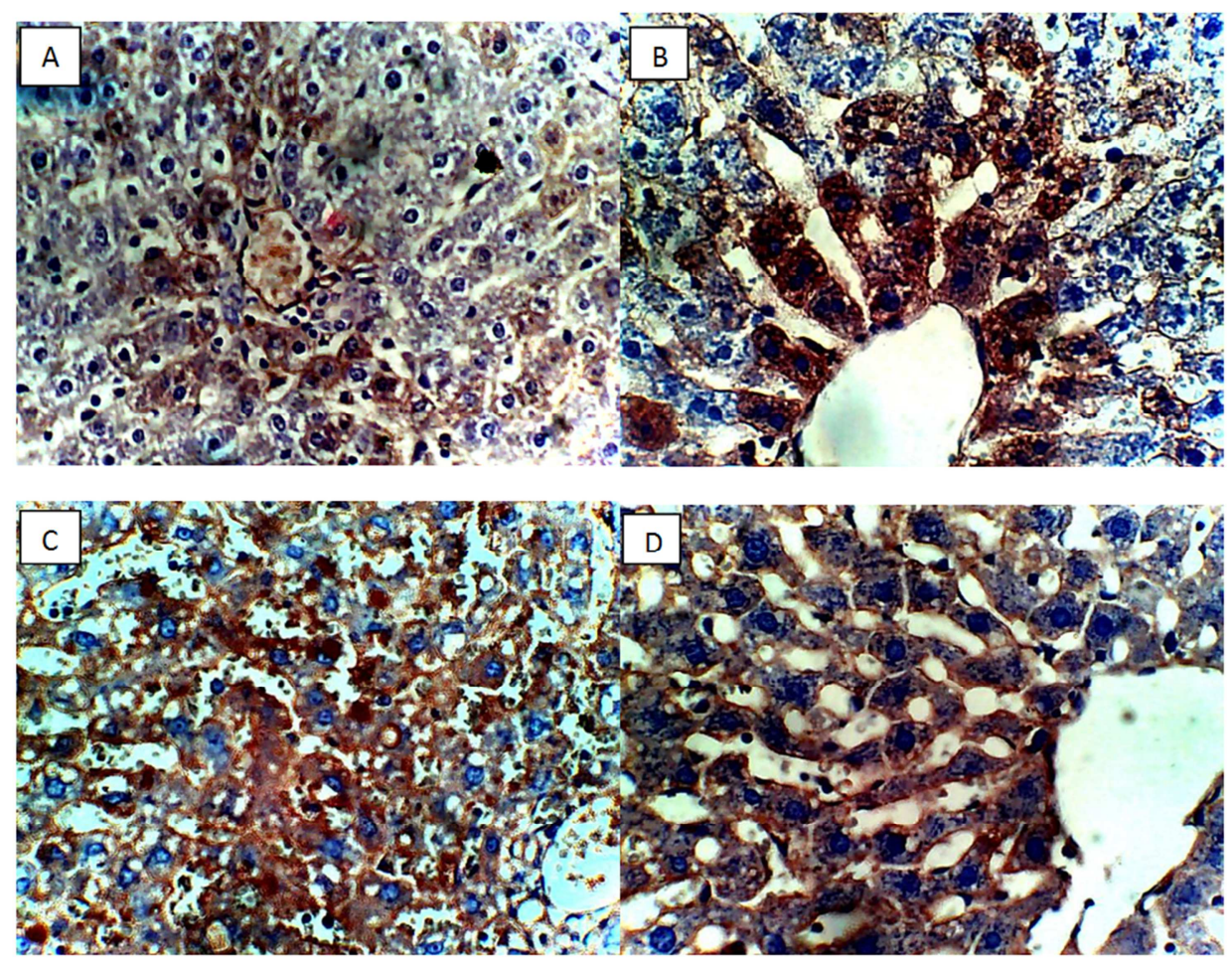

Figure 2. $(A, B, C, D): 2 A$; Micrograph showing the expression of CEA of liver from $1 \mathrm{ml}$ distill water treated rat $x$ 400. $2 B$; Micrograph showing the expression of CEA of liver from $9.29 \mathrm{mg} / \mathrm{kg}$ body weight of Douvir-N treated rat x 400. 2C; Micrograph showing the expression of CEA of liver from 9.29mg/kg body weight of Douvir-Nand $0.07 \mathrm{mg} / \mathrm{kg}$ of folic acid $\times 400.2 \mathrm{D}$; Micrograph showing the expression of CEA of liver from $0.07 \mathrm{mg} / \mathrm{kg}$ body weight of folic acid treated rat $x 400$. 

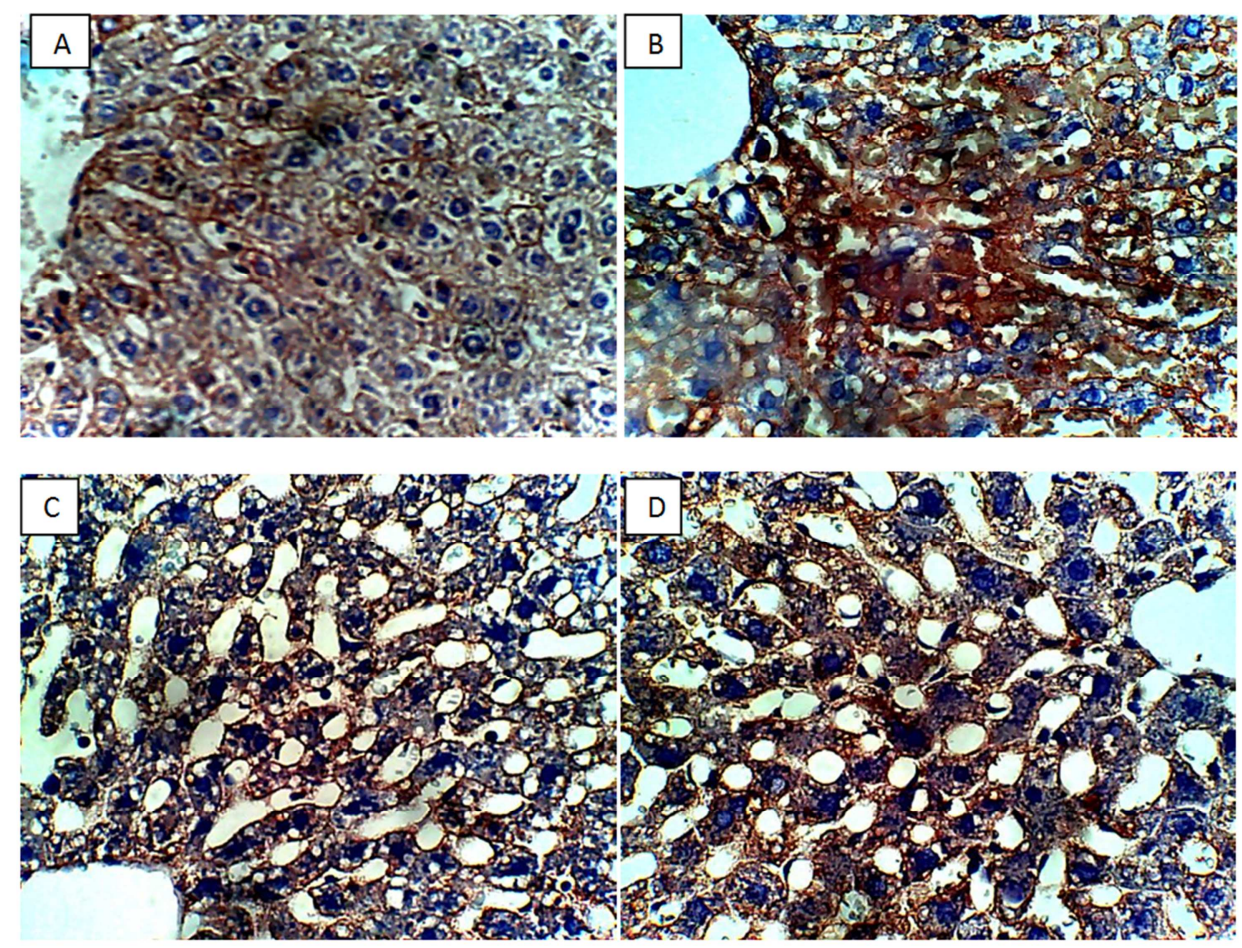

Figure 3. $(A, B, C, D)$ : $3 A$; Micrograph showing the expression of $C K-7$ of liver from $1 \mathrm{ml}$ distill water treated rat $x$ 400. 3B; Micrograph showing the expression of CK-7 of liver from $9.29 \mathrm{mg} / \mathrm{kg}$ body weight of Douvir-N treated rat x 400. 3C; Micrograph showing the expression of CK-7 of liver from $9.29 \mathrm{mg} / \mathrm{kg}$ body weight of Douvir-Nand $0.07 \mathrm{mg} / \mathrm{kg}$ of folic acid treated rat $x 400$. 3D; Micrograph showing the expression of CK-7 of liver from $0.07 \mathrm{mg} / \mathrm{kg}$ body weight of folic acid treated rat $x 400$.

Table 1. Levels of ast), alt and alp in the serum of wistar rats.

\begin{tabular}{llll}
\hline \multirow{2}{*}{ Group(s) } & \multicolumn{3}{l}{$\begin{array}{l}\text { The level of these biomarkers were expressed as Mean } \pm \\
\text { SEM }\end{array}$} \\
\cline { 2 - 4 } & AST & ALT & ALP \\
\hline A & $78.20 \pm 10.29$ & $34.80 \pm 1.83$ & $89.00 \pm 5.14$ \\
B & $111.00 \pm 11.24$ & $46.20 \pm 3.75^{*}$ & $130.60 \pm 2.79$ \\
C & $93.00 \pm 6.47$ & $41.80 \pm 1.56^{*}$ & $101.20 \pm 6.58$ \\
D & $76.40 \pm 5.95$ & $39.20 \pm 3.57$ & $106.60 \pm 6.60$ \\
\hline
\end{tabular}

* = significant difference from control group $(\mathrm{P}<0.05)$

\section{Discussion}

Highly active antiretroviral therapy (HAART) has been associated with toxicities including those affecting the liver [25]. Drugs are important cause of liver injuries. More than 900 drugs, toxins, and herbs have been reported to cause liver injury, and drugs account for $20-40 \%$ of all instances of fulminant hepatic failure [26]. Knowledge of the commonly implicated agents and a high index of suspicion are essential in diagnosis. This study was designed to investigate the effects of Douvir-N and its coadministration with folic acid.

The results obtained from this study revealed that oral administration of Douvir-N had toxic effects on the liver, with moderate distortion of liver cellular architecture with dilatation of the central vein, sinusoidal spaces and pyknotic nuclei changes. These changes were supported by immunohistochemical findings which revealed increased expression of CEA and CK-7 suggestive of liver damage. CEA is a non specific marker for cancers and liver inflammation caused by hepatitis or chemotherapy [27] it is also useful in the evaluation of cancer [27], and gastric inflammatory changes which suggest a close relationship between gastric CEA values and the degree of gastric inflammation [28]. The study of cytokeratin expression has provided a valuable insight into the biliary microanatomy of the liver in health and disease. A study has shown increased expression of CK-7 in liver disease [29].

This has serious implications for patients on antiretroviral therapy as a functional liver is needed for metabolism of drugs, production of bile and storage of glycogen. Drug related injury can further deteriorate the health of the patients. This can leads to poor medication adherence and ultimate virological failure and death [30].

In general, severe hepatic injuries have been documented to occur in HAART patients, regardless of their treatment [31]. ALT and AST are liberated into the blood whenever liver cells are damaged and increased plasma enzymes activity is a sensitive index of hepatic damage [32, 33]. Neither of these enzymes is specific to the liver but ALT occurs in much higher concentration in the liver than elsewhere [33]. Therefore, the increased serum ALT activity in the groups that were administered with Douvir-N in this study more specifically reflects hepatic damage. This agreed with the histological findings which revealed liver distortions. Mechanism of liver injury due to ARV is poorly understood; Hypersensitivity and mitochondrial damage have been shown to contribute to these injuries [34] and these might have been the mechanism of liver injury in this study.

These changes were reduced in the groups that Douvir-N 
was administered with folic acid, as shown in the reduction in liver enzymes, the mild expression of CEA and CK-7 immuno-markers and the improved architecture of the sinusoidal spaces, hepatocytes and nuclei in the H/E sections. This implies that folic acid could provide cytoprotection to the liver of the people taking Douvir-N and this can lead to improvement in the health of people taking this antiretroviral drug.

In conclusion administration of oral doses of Douvir- $\mathrm{N}$ is harmful to the histochemistry of the liver, leading to increased expression of CEA, CK-7 and increased ALT level. There was a slight ameliorative effect when co-administered with folic acid. Folic acid on its own did not produce any harmful effects on the liver. It is therefore expedient to carry out further studies on this great potential of folic acid with a view to subsequently recommending it to patients on Douvir-N therapy.

\section{References}

[1] https://www.patientslikeme.com/treatment evaluations/brows e/20908-douvirn-reviews? brand=f. Retrieved on line March $22^{\text {nd }}, 2015$

[2] http://www.aidsmeds.com/archive/PIs_1068.shtml. Retrieved online on June $7^{\text {th }} 2014$.

[3] http://www.globalsources.com/si/AS/Parthweb-Solutions/600 8837110868/pdtl/Duovir-N /1116459774.htm. Retrieved on line March $22^{\text {nd }}, 2015$.

[4] Schambelan, M., Benson, C. and Carr, A. Acquired immune deficiency syndrome. Int. AIDS Soc. USA. Panel, 2002; 9: 30-32.

[5] http://www.indiamart.com/shivroyallifecare/cipla-hiv.html. Retrieved online on March 22nd, 2015.

[6] http://aidsinfo.nih.gov/contentfiles/sideeffectanithivmeds_cbr ochure_en.pdf retrieved online on February 11th, 2015.

[7] Sulkowski, M. S., Thomas, D. L., Mehta, S. H., Chaisson, R.E and Moore, D. R. Hepato-toxicity associated with nevirapine or efavirenz-containing antiretroviral therapy: Role of hepatitis C and B infections. Hepatology, 2002; 35: 182-189.

[8] Abrescia, N., M. D'Abbraccio, M. Figoni, A. Busto, A. Maddaloni and De Marco, M. Hepatotoxicity of antiretroviral drugs. Curr. Pharm. Des., 2005; 11: 3697-3710.

[9] Verucchi, G. L., Calza, L., Biagetti, C., Attard, L., Costagliola, R., Manfredi, R. et al. Ultrastructural liver mitochondrial abnormalities in $\mathrm{HIV} / \mathrm{HCV}$-coinfected patients receiving antiretroviral therapy. J Acquir Immune Defic Syndr. 2004; 35: 326-328.

[10] Walker, U. J. Depletion of mitochondrial DNA in liver under antiretroviral therapy with didanosine, stavudine, or zalcitabine. Hepatology, 2004; 39: 311-317.

[11] Carr, A., Morey, A., Mallon, P., Williams, D. and Thorburn, D. R Fatal Portal Hypertension, Liver Failure, and Mitochondrial Dysfunction after HIV-1 Nucleoside Analogue-Induced Hepatitis and Lactic Acidaemia. The Lancet, 2001; 357(9266): $1412-1414$
[12] Glenn, J. and Adam, I. Neurologic and Psychiatric Complications of Antiretroviral Agents. AIDS, 2002; 16:1201-1215.

[13] McDowell, M. A., Lacher, D. A and Pfeiffer, C. M. Blood folate levels: the latest NHANES results. NCHS data briefs, 2008; 6:1-8.

[14] Berry, R. S; Li, Z and Erickson, J. D. Prevention of NTD with folic acid in china. US collaborative project for NTD prevention. N. Engl. J. med, 1999; 341: 1485-90.

[15] Botto, L. D; Moore, C. A and Khoury, M. J. Neural tube defects. N. Engl. J. med. 1999; 34:1509-19.

[16] Hobbins, J. C. Diagnosis and management of NTDs today. N. Engl. J. Med. 1991; 324:690-91.

[17] Kim, Y. I. Role of folate in cancer development and progression. J. Nutrition. 2003; 133 (11); 3731-3739.

[18] Boushey, C. J., Beresford, S. A., Omenn, G. S and Motulusky, A. G. A quantitative assessment of plasma homocysteine as a risk for vascular disease: probable benefits of increasing folic acid intakes. JAMA. 1995; 274: 1049-1057.

[19] Ebisch, I. M., Thomas, C. M., Peters, W. H., Braat, D. D and Steegers-Theunissen, R. P. the importance of folate, zinc and antioxidants in the pathogenesis and prevention of subfertility. Hum. Reprod Update. 2007; 13:163-174.

[20] Mason, J. B. “A temporal association between folic acid fortifications and an increase in colorectal cancer rates may be illuminating important biological principles: a hypothesis". Cancer Epidemiol Biomarker Prev. 2007; 16 (7): 1325-1329.

[21] Doshi, S. N., McDowell, I. F., Moat, S. J., Land, D., New Combe, K. G., Kredan, M. B. et al. Folate improves Endothelial function in coronary artery disease: an effect mediated by Reduction in intracellular superoxide. Arterioscler Thromb vasc Biol. 2001; 21: 196-1202.

[22] Patrick, H. The Prevention of Memory Loss and Progression to Alzheimer's Disease with B Vitamins, Antioxidants and Essential Fatty Acids: A Review of the Evidence. Available at http://www.foodforthebrain.org/media/229772/Holford.pdf. retrieved August 20th, 2015.

[23] Bryan, J, Calvaresi, E and Hughes, D. Short term folate, vitamin B12 supplementation slightly affects memory performance but not mood of women in various ages. J. Nutr. $2002 ; 132,1345-1356$.

[24] Gautam, N., Puligujja, P., Balkundi, S., Thakare, R., Liu, X., and Fox, H. et al. Pharmacokinetics, Biodistribution, and Toxicity of Folic Acid-Coated Antiretroviral Nanoformulations. Antimicrob Agents Chemother. 2014 Dec; 58 (12): 7510-7519.

[25] Moreno-Cuerda, V. J, Morales-Conejo, M and Rubio, R "Antiretroviral treatment associated life-threatening adverse events", Medicina clinica, 2006; 126 (19): 744-749.

[26] http://emedicine.medscape.com/article/169814-overview retrieved August 20th, 2015.

[27] http://www.medicinenet.com/carcinoembryonic_antigen/articl e.htm retrieved online on the $6^{\text {th }}$ of August 2015.

[28] Micali B, Florio MG, Venuti A, Artemisia A, Caputo G, Brancato U. Usefulness of carcinoembryonic antigen measurement in gastric juice of patients with gastric disorders. J Clin Gastroenterol. 1983; 5(5):411-5. 
[29] Bateman, A. C. and Hübscher, S. G. Cytokeratin expression as an aid to diagnosis in medical liver biopsies Histopathology 2010, Volume 56, Issue 4, pages 415-425.

[30] Castelnuovo B, John L, Lutwama F, Ronald A, Spacek L. A, Bates M, et al. "Three-year outcome data of second-line antiretroviral therapy in Ugandan adults: good virological response but high rate of toxicity," Journal of the International Association of Physicians in AIDS Care, 2009; vol. 8, no. 1, pp. $52-59$.

[31] M. Núñez, R. Lana, J. L. Mendoza, L. Martín-Carbonero, and V. Soriano, "Risk factors for severe hepatic injury after introduction of highly active antiretroviral therapy," Journal of Acquired Immune Deficiency Syndromes, , 2001; vol. 27, no. 5, pp. $426-431$.

[32] Edwards, C. R. W., Bouchier, I. A. D., Haslett, C. and Chilvers, E. E. Diabetes Mellitus in Davidson's Principle and Practice of Medicine (10th Edition). Churchhill Livingstone, London. 2008; 724-774.

[33] Crook, M. A. Clinical Biochemistry and metabolic Medicine. $8^{\mathrm{TH}}$ ed. Edwaed Arnold publishers, London, 2012; 254-255.

[34] http://telemedicine.itg.be/telemedicine/Uploads/ARLI_CME.p df retrieved online on the $26 / 8 / 2015$. 